\title{
Liver resection versus chemoembolization for patients with multifocal hepatocellular carcinoma
}

\author{
Victor M. Zaydfudim, Reid B. Adams \\ Department of Surgery, Section of Hepatobiliary and Pancreatic Surgery, University of Virginia, Charlottesville, VA, USA \\ Correspondence to: Victor M. Zaydfudim, MD, MPH. Department of Surgery, Section of Hepatobiliary and Pancreatic Surgery, Box 800709, \\ University of Virginia, Charlottesville, VA, USA. Email vz8h@virginia.edu. \\ Comment on: Fukami Y, Kaneoka Y, Maeda A, et al. Liver Resection for Multiple Hepatocellular Carcinomas: A Japanese Nationwide Survey. Ann \\ Surg 2019. [Epub ahead of print].
}

Submitted Apr 05, 2019. Accepted for publication Apr 16, 2019.

doi: 10.21037/hbsn.2019.04.12

View this article at: http://dx.doi.org/10.21037/hbsn.2019.04.12

Curative intent treatment strategies for patients with hepatocellular carcinoma (HCC) include liver resection, liver transplantation, and arguably tumor ablation. Locoregional therapies including transarterial chemoembolization (TACE) as well as transarterial radioembolization (TARE) remain viable treatment options for patients who are not candidates for therapy with curative intent or patients awaiting liver transplantation receiving downstaging treatment as part of intention to proceed with transplantation. A number of published single institution studies and pooled analyses provide data demonstrating improved survival in patients with HCC treated with resection rather than TACE $(1,2)$. These data support liver resection rather than TACE in appropriately selected patients with multifocal HCC. The recently published analysis by Fukami et al. for the Liver Cancer Study Group of Japan provides robust evidence demonstrating greater survival in patients with multifocal HCC ( $\leq 3$ tumors) selected for liver resection rather than TACE (3).

This study reports findings from a retrospective Japanese Nationwide Survey of 3,246 patients with Child-Pugh A cirrhosis and multifocal HCC ( $\leq 3$ tumors) who were treated between 2000 and 2007. From this cohort, liver resection was chosen as the first line therapy in 1,944 patients and 1,302 patients were treated with TACE. This is the largest published analysis with granular data comparing liver resection to TACE in patients with multifocal HCC. The dataset and analyses are robust; perioperative outcomes are outstanding with $0.57 \% 90$-day mortality. Both full cohort and propensity matched analyses demonstrate greater overall and disease-free survival among patients selected for liver resection rather than TACE. Resection-first treatment consideration appears to dominate as more patients with multifocal HCC in the overall cohort were selected for resection rather than TACE. While it is difficult to ascertain retrospectively, patients with older age, poor liver function or reserve (with surrogate metrics such as low albumin, higher bilirubin, low platelets, higher ICG15 retention) and more tumors (reported as proportion of patients with 3 tumors compared to 2) were more likely to have been selected for TACE rather than resection. However, both propensity matched analyses and a multivariate model, demonstrate greater survival in patients selected for liver resection than TACE. Known risk factors such as older age, low serum albumin, high AFP, macrovascular invasion, and larger tumor size were all independently associated with lower overall survival after multivariable adjustment.

Importantly, survival statistics from this multi-institutional Japanese series, demonstrate overall 5-year survival approaching $60 \%$ among patients selected for liver resection and $42 \%$ among patients selected for TACE. Five-year disease-free survival approximated 34\% after liver resection and $23 \%$ after TACE. Results of this study underscore the safety of liver resection performed in appropriately selected patients with multifocal (but $\leq 3$ tumors) HCC and confirm greater survival in patients selected for liver resection rather than TACE.

Is liver resection the optimal treatment for multifocal HCC? A number of published analyses support liver transplantation rather than liver resection as the best 
treatment for cirrhotic patients with multifocal HCC both within as well as beyond the Milan criteria $(4,5)$. However, numerous patient specific, organ allocation, and societal/ cultural beliefs influence availability and utilization of liver transplantation among patients with HCC (6). While some factors in selection for transplantation might be modified (e.g., patient selection parameters or greater use of livingdonor or split-liver organs), others (e.g., socioeconomic barriers or societal/cultural norms) are considerably more difficult to influence and change. Additionally, while the role of transplantation continues to grow with an increase in available donor organs and techniques, liver resection remains the primary curative treatment option for patients not eligible for transplantation.

To this extent two emerging advances in HCC treatment are of interest. From a patient selection and operative consideration perspective, salvage liver transplantation, albeit still not common, is growing in popularity as a possible curative treatment in patients with HCC recurrence after resection $(7,8)$. Intention-to-treat comparisons continue to demonstrate greater survival with upfront transplantation, however, patients who were able to complete salvage transplantation after recurrence had similar survival statistics compared to primary liver transplant recipients. In theory, most patients could be candidates for salvage transplantation, however only a minority of patients in most transplant centers undergo transplantation for HCC recurrence. At centers pursuing an active salvage transplantation strategy, patient (MELD) and tumor (absence of microvascular invasion) factors have been associated with improved success of both salvage transplantation and survival.

Advances in systemic therapy are improving and worth considering. While, at present, locoregional treatment continues to provide more durable tumor control than systemic therapy, expansion of targeted therapy and immunotherapy options have allowed for additional treatment options in patient with multifocal advancedstage HCC $(9,10)$. Two kinase inhibitors (lenvatinib and sorafenib) are currently approved as first line therapy for patients with advanced-stage disease and the number of second-line targeted therapies continues to increase. Immunotherapy has revolutionized medical therapy for a number of solid organ malignancies including lung, head and neck, renal cancers and melanoma. A number of immune check-point inhibitors and combination therapies are currently in Phase III clinical trials in patients with advanced-stage HCC.

Treatment options for patients with multifocal HCC continue to grow. A multi-disciplinary treatment approach is paramount. Liver transplantation should be considered as first line therapy in patients eligible for transplantation where transplantation is available. The recently published Fukami et al. study (3) supports liver resection as the next best treatment option in patients with Child-Pugh A preserved liver function and multifocal HCC not exceeding 3 tumors. A combination of tumor resection and ablation could be considered for patients with cirrhosis and multifocal disease including individual tumors not amenable to parenchymal sparing resections. Locoregional and systemic therapy remain options for patients with multifocal HCC who are not candidates for curative-approach treatment.

\section{Acknowledgments}

Funding: This work was supported by the L30 CA220861 Award from National Cancer Institute and Grant \#IRG 81-001-26 from the American Cancer Society to VM Zaydfudim .

\section{Footnote}

Conflicts of Interest: The authors have no conflicts of interest to declare.

\section{References}

1. Cucchetti A, Mazzaferro V, Pinna AD, et al. Average treatment effect of hepatic resection versus locoregional therapies for hepatocellular carcinoma. Br J Surg 2017;104:1704-12.

2. Liang L, Xing $H$, Zhang $H$, et al. Surgical resection versus transarterial chemoembolization for BCLC intermediate stage hepatocellular carcinoma: a systematic review and meta-analysis. HPB (Oxford) 2018;20:110-9.

3. Fukami Y, Kaneoka Y, Maeda A, et al. Liver Resection for Multiple Hepatocellular Carcinomas: A Japanese Nationwide Survey. Ann Surg 2019. [Epub ahead of print].

4. Chapman WC, Klintmalm G, Hemming A, et al. Surgical treatment of hepatocellular carcinoma in North America: can hepatic resection still be justified? J Am Coll Surg 2015;220:628-37.

5. Zaydfudim VM, Vachharajani N, Klintmalm GB, et al. Liver resection and transplantation for patients with hepatocellular carcinoma beyond milan criteria. Ann Surg 2016;264:650-8.

6. Zaydfudim VM, Chapman WC, Nagorney DM. 
Challenges in patient selection for liver resection or transplantation in patients with hepatocellular carcinoma beyond Milan criteria. Hepatobiliary Surg Nutr 2017;6:287-9.

7. Bhangui P, Allard MA, Vibert E, et al. Salvage Versus Primary Liver Transplantation for Early Hepatocellular Carcinoma: Do Both Strategies Yield Similar Outcomes? Ann Surg 2016;264:155-63.

8. de Haas RJ, Lim C, Bhangui P, et al. Curative Salvage Liver Transplantation in Cirrhotic patients with

Cite this article as: Zaydfudim VM, Adams RB. Liver resection versus chemoembolization for patients with multifocal hepatocellular carcinoma. HepatoBiliary Surg Nutr 2019;8(5):543545. doi: 10.21037/hbsn.2019.04.12
Hepatocellular Carcinoma: An intention-to-treat analysis. Hepatology 2018;67:204-15.

9. Llovet JM, Montal R, Sia D, et al. Molecular therapies and precision medicine for hepatocellular carcinoma. Nat Rev Clin Oncol 2018;15:599-616.

10. Obeid JM, Kunk PR, Zaydfudim VM, et al. Immunotherapy for hepatocellular carcinoma patients: is it ready for prime time? Cancer Immunol Immunother 2018;67:161-74. 